\title{
External evaluation of the Briganti nomogram to predict lymph node metastases in intermediate-risk prostate cancer patients
}

Running title: External evaluation of the Briganti nomogram

Nicolas Peilleron ${ }^{a}$, Arnaud Seigneurin ${ }^{b, d}$, Caroline Herault ${ }^{d}$, Camille Verry ${ }^{c}$, Michel Bollac, Jean-Jacques Rambeaud a , Jean-Luc Descotes ${ }^{a, b}$, Jean-Alexandre Long ${ }^{a, b}$, Gaelle Fiard ${ }^{a, b}$

a Department of Urology, Grenoble Alpes University Hospital, Grenoble, France

b Univ. Grenoble Alpes, CNRS, Grenoble INP, TIMC IMAG, Grenoble, France

' Department of Radiotherapy, Grenoble Alpes University Hospital, Grenoble, France

${ }^{\mathrm{d}}$ Department of Medical assessment, Grenoble Alpes University Hospital, Grenoble, France

Corresponding author:

Gaelle Fiard

Department of urology

Grenoble Alpes University Hospital

CS10217

38043 Grenoble Cedex 9

France

Email: gfiard@chu-grenoble.fr

ORCID: 0000-0003-3049-5318

Keywords

Prostate cancer, lymph node dissection, nomogram, radical prostatectomy

Word count: 2906

\section{Abstract}

Purpose: The Briganti nomogram can be used with a threshold of $5 \%$ to decide when to offer lymph node dissection during radical prostatectomy. The objective of the study was to assess the accuracy of the Briganti nomogram on intermediate-risk prostate cancer patients managed in a single academic department.

Methods: We retrospectively reviewed the files of all patients managed by radical prostatectomy (RP) and bilateral pelvic lymph node dissection (BPLND) in our center between 2005 and 2017. The overall accuracy of the model in predicting metastatic lymph node disease was quantified by the construction of a receiver-operator characteristic (ROC) curve. A 
calibration plot was drawn to represent the relationship between the predicted and observed frequencies.

Results: We included 285 patients, among whom 175 (61.4\%) were classified as intermediate risk as defined by D'Amico. The median follow-up was 60 (34-93) months. Twenty-seven patients (9.5\%) were diagnosed with lymph node metastases. The median number of lymph nodes removed was 10 (7-14). The mean Briganti score was $19.3 \%$ in patients with lymph node involvement (LNI) and 6.3\% in patients without LNI. Focusing on intermediate-risk patients, 91(52\%) and 84 (48\%) had a Briganti score $<5 \%$ and $\geq 5 \%$, respectively, among whom 6 (6.6\%) and $7(8.3 \%)$ had lymph node metastases. The accuracy of the score was low for intermediate risk patients with an area under the curve (AUC) of 53.1\% (95\% Cl 0.45-0.61).

Conclusion: The Briganti nomogram in our retrospective cohort showed low accuracy for the prediction of lymph node involvement in an intermediate-risk prostate cancer population.

Keywords: prostate cancer, nomogram, staging, lymph node involvement, radical prostatectomy

\section{Introduction}

Thanks to the widespread use of PSA screening, over $90 \%$ prostate cancer (PCa) cases are nowadays diagnosed at a localized stage. Among these patients, using the D'Amico classification, around $25 \%$ of the patients are in the intermediate-risk group [1].

Radical prostatectomy (RP) and external-beam radiation therapy (EBRT) combined with short-term androgen-deprivation therapy (ADT) are the two standard radical treatment options for this group of patients [2,3]. Nowadays, when RP is chosen, the EAU guidelines recommend the performance of extended bilateral pelvic lymph node dissection (eBPLND) if the risk of lymph node metastases is greater than $5 \%[4,5]$.

The probability of lymph node involvement (LNI) can be estimated via the use of nomograms including that of Briganti and al $[6,7]$. It was initially developed and recently validated in high-risk patients to identify those who would benefit the most from RP or EBRT with short-term ADT based on their lymph node status $[8,9]$.

Intermediate-risk patients are probably the most heterogeneous group. The lymph node metastases rate within this group may range from $3.7 \%$ to $20.1 \%$ [7]. Although the 
eBPLND is the gold standard for nodal staging, it is associated with a significant morbidity rate up to $19.8 \%$ [10]. The use of nomograms therefore aims at selecting patients at a higher risk of LNI to reduce morbidity. However, nomograms are usually developed from a very selected cohort of patients and can prove less appropriate in different settings.

Our primary objective was to evaluate the accuracy of the Briganti score (SB) in predicting the risk of LNI in a cohort of intermediate-risk patients treated with RP and BPLND. Our secondary objectives were to explore potential predictive factors of nomogram inaccuracy and lymph node metastases, to report the biochemical recurrence-free survival of patients with or without LNI, and to assess the consequences of the systematic application of the nomogram using various thresholds to our cohort.

\section{Material and Methods}

\section{Patients and methods}

We retrospectively collected perioperative and follow-up data of consecutive patients treated by RP and BPLND for localized prostate cancer in our center between 2005 and 2017.

Preoperative data included PSA level, clinical stage, Gleason score and percentage of positive biopsies. The clinical stage was determined according to the cTNM 2002 classification updated in 2010. Prostate biopsies were performed according to the currently recommended regimen of 12 randomized, ultrasound-guided biopsies, with the use since 2011 of an MRIultrasound fusion system (Koelis, France) allowing the realization of targeted biopsies in addition. The Gleason score was determined on biopsies and the prostatectomy specimen by pathologists specialized in urology, according to the grading system defined by Gleason in 1966 and reviewed by the ISUP (International Society of Urological Pathology) in 2005 and 2014. Pathologists reported the pathological stage with reference to the 2002 pTNM classification updated in 2009.

All patients had a preoperative pelvic MRI to assess lymph node metastatic status.

The Briganti score was calculated for all patients using the 2012 version. PSA dosage was obtained 4-6 weeks after surgery. Persistent detectable PSA was defined by a dosage > $0.2 \mathrm{ng} / \mathrm{ml}$.

Open, laparoscopic and robot assisted (using the DaVinci Si system) laparoscopic RP were performed by senior and junior surgeons. A standard extended BPLND template was 
used including external, internal iliac up to the ureteral crossing, and obturator lymph nodes. The learning curve of the supervising surgeon performing the robotic procedures started in 2014 and ended in 2016, based on procedure duration. Complications related to BPLND were noted and rated using the Clavien classification.

The study received institutional review board approval and was registered with the CNIL reference number: 2216943v0 (French National Agency regulating Data Protection).

\section{Follow-up}

All patients were reviewed in outpatient clinic 6 weeks after surgery with a first PSA dosage. In case of undetectable PSA, new appointments were scheduled every 6 months during the first 2 years of follow-up, then on a yearly basis up to 5 years, with PSA dosage still performed on a 6-monthly basis. Patients were then usually referred back to their GP with a recommendation of annual PSA testing provided that the PSA had remained undetectable.

Biochemical recurrence (BCR) was defined by a PSA level $>0.2 \mathrm{ng} / \mathrm{ml}$ confirmed on 2 successive postoperative dosages. The threshold was set at $0.4 \mathrm{ng} / \mathrm{ml}$ when adjuvant radiotherapy was performed.

Patients with LNI and undetectable post-operative PSA were given the option of surveillance and early salvage treatment in case of rising PSA values with salvage radiotherapy and concomitant androgen deprivation therapy. In case of detectable post-operative PSA, adjuvant radiotherapy with a dose of $66 \mathrm{~Gy}$ and optional concomitant androgen deprivation therapy was offered. All cases were discussed in a dedicated multidisciplinary meeting.

\section{Statistical analysis}

Continuous variables were reported using median values with interquartile ranges (IQR) and compared with the Kruskal-Wallis test. Categorical variables were reported as counts and proportions (\%) and compared using the chi-2 test or Fisher's exact test, as appropriate.

The accuracy of the Briganti score was evaluated using Receiver Operating Characteristics (ROC) curves, analyzed by calculating the Area Under Curve (AUC). The AUC tells us about the discrimination of the model, i.e. its ability to distinguish patients with LNI and those without LNI. We also used a calibration curve, representing the relation between 
the predicted frequencies on the $x$-axis and the observed frequencies on the $y$-axis. Calibration is the concordance between the predictions and the observations of the model. If the model predicts perfectly, all points must be on a $45^{\circ}$ line. Each point corresponds to a group of patients for which the observed proportion of LNI and the probability predicted by the model were calculated.

We performed two multivariate analyses, using logistic regression, for estimating adjusted Odds Ratio on potential predictive factors of lymph node metastases and Briganti score inaccuracy.

BCR-free survival rates were calculated from the date of surgery, which was the date of origin, to the date of diagnosis of BCR or death or last follow-up. Survival curves were performed according to the Kaplan-Meier method, and comparison between groups using the log-rank test.

All reported $p$-values were two-sided. Significance level was defined at 0,05 for all tests. The Bonferroni correction was applied for the multiple tests performed to limit the risk of alpha risk inflation. In our study 19 tests were performed, which explains a significance level set at 0.002632 . Statistical analyses were performed with STATA ${ }^{\circledR} 16.0$ and $R^{\circledR} 3.6 .1$ softwares.

\section{Results}

\section{Population and surgery outcomes}

Between 2005 and 2017, 285 patients were treated by RP and BPLND for localized prostate cancer in our center. Among them, 175 patients were in the intermediate-risk group. Patient characteristics are detailed in Table 1.

Lymph node involvement was present among $7.4 \%(\mathrm{~N}=13)$ of intermediate-risk patients and $9.5 \%(\mathrm{~N}=27)$ of all patients.

The rate of positive surgical margins was $36.6 \%(\mathrm{~N}=64)$ in the intermediate group and $38.6 \%(N=110)$ for the entire cohort.

After a median follow-up of 60 (34-93) months, the rate of BCR was $30.9 \%(\mathrm{~N}=88)$ and was higher in the group with LNI $(66.7 \%(N=18)$ vs $27.1 \%(N=58) ; p<0.001)$. 
Table 1. Patient characteristics

\begin{tabular}{|c|c|c|}
\hline & $\begin{array}{c}\text { Intermediate-risk } \\
\text { patients }(\mathrm{N}=175)\end{array}$ & $\begin{array}{c}\text { All patients } \\
(\mathrm{N}=\mathbf{2 8 5})\end{array}$ \\
\hline Median (IQR) age, years & $65(61-69)$ & $65(61-69)$ \\
\hline Median (IQR) PSA, ng/ml & $8(5.6-11.1)$ & $7.9(5.6-11.0)$ \\
\hline \multicolumn{3}{|l|}{ Clinical stage, $\mathbf{N}(\%)$} \\
\hline $\mathrm{T} 1$ & $81(46.3)$ & $124(43.5)$ \\
\hline $\mathrm{T} 2$ & $94(53.7)$ & $156(54.7)$ \\
\hline T3 & $0(0)$ & $5(1.8)$ \\
\hline Extraprostatic extension on MRI, N (\%) & $32(18.3)$ & $66(23.2)$ \\
\hline Median (IQR) \% positive biopsies & $33.3(16.7-50.0)$ & $33.3(16.7-50.0)$ \\
\hline \multicolumn{3}{|l|}{ Biopsy primary Gleason score, N (\%) } \\
\hline$<=3$ & $113(64.6)$ & $178(62.5)$ \\
\hline$>=4$ & $62(35.4)$ & $107(37.5)$ \\
\hline \multicolumn{3}{|l|}{ Biopsy secondary Gleason score, N (\%) } \\
\hline$<=3$ & $86(49.1)$ & $150(52.6)$ \\
\hline$>=4$ & $89(50.9)$ & $135(47.3)$ \\
\hline Briganti Score >= 5\%, N (\%) & $84(48.0)$ & $144(50.5)$ \\
\hline Mean Briganti Score, \% & 6 & 7.5 \\
\hline \multicolumn{3}{|l|}{ Prostatectomy Gleason score, N (\%) } \\
\hline$<7$ & $23(13.1)$ & $46(16.1)$ \\
\hline 7 & $141(80.6)$ & $202(70.9)$ \\
\hline$>7$ & $10(5.7)$ & $37(13.0)$ \\
\hline \multicolumn{3}{|l|}{ Pathological stage, N (\%) } \\
\hline pT0 & $1(0.6)$ & $1(0.3)$ \\
\hline pT2 & $106(60.6)$ & $164(57.5)$ \\
\hline pT3 & $67(38.3)$ & $118(41.4)$ \\
\hline pT4 & $1(0.6)$ & $2(0.7)$ \\
\hline $\begin{array}{c}\text { Surgical margin, } \mathbf{N}(\%) \\
\text { RO } \\
\end{array}$ & $111(63.4)$ & $175(61.4)$ \\
\hline $\mathrm{R} 1$ & $64(36.6)$ & $110(38.6)$ \\
\hline Number of nodes removed, median (IQR) & $9(7-14)$ & $10(7-14)$ \\
\hline Lymph node involvement, $\mathrm{N}(\%)$ & $13(7.4 \%)$ & $27(9.5 \%)$ \\
\hline Post-operative undetectable PSA, N (\%) & $164(93.7)$ & $257(90.8)$ \\
\hline Adjuvant treatment, $\mathrm{N}(\%)$ & $15(8.6)$ & $36(12.6)$ \\
\hline All complications, $\mathbf{N}(\%)$ & $19(10.9)$ & $26(9.1)$ \\
\hline Clavien $\geq 3$ & $10(5.7)$ & $13(4.6)$ \\
\hline Biochemical recurrence, $\mathbf{N}(\%)$ & $49(28.0)$ & $88(30.9)$ \\
\hline
\end{tabular}

The rate of complications related to BPLND was $10.9 \%(\mathrm{~N}=19)$ in the intermediate group and $9.1 \%(\mathrm{~N}=26)$ in the entire cohort. Among these $4.6 \%(\mathrm{~N}=13)$ were rated Clavien 3 or greater (lymphocele requiring drainage or surgery $n=9$; ureteral injury $n=2$; iliac injury with hemorrhagic shock $n=1$; iliac thrombosis with massive pulmonary embolism and death $n=1$ ). 


\section{Accuracy of the Briganti score}

ROC curve analysis (Figure 1) respectively showed low and fairly relevant accuracy of the score for intermediate risk patients (AUC 53.1\%, 95\% Cl 0.45-0.61) (Fig 1a) and all patients (AUC 75.2\%, 95\% Cl 0.7-0.8) (Fig 1b), respectively. The calibration plot revealed a good correlation between the predicted probabilities of the score on the $\mathrm{x}$ axis and the observed proportion of LNI on the y axis across the $45^{\circ}$ slope (Figure 2 ).

Figure 1. ROC and area under curve

1a. Intermediate-risk patients $(\mathrm{N}=175)$ : AUC $=0.531$

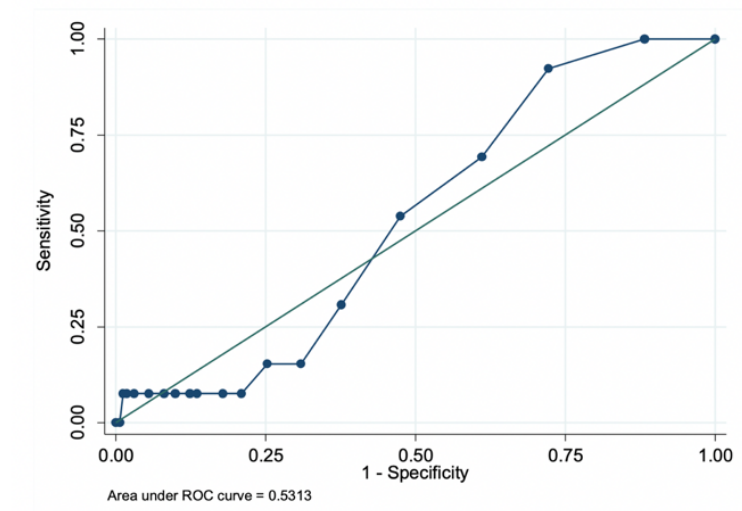

1b. All-risk patients ( $\mathrm{N}=\mathbf{2 8 5})$ : AUC $=0.752$

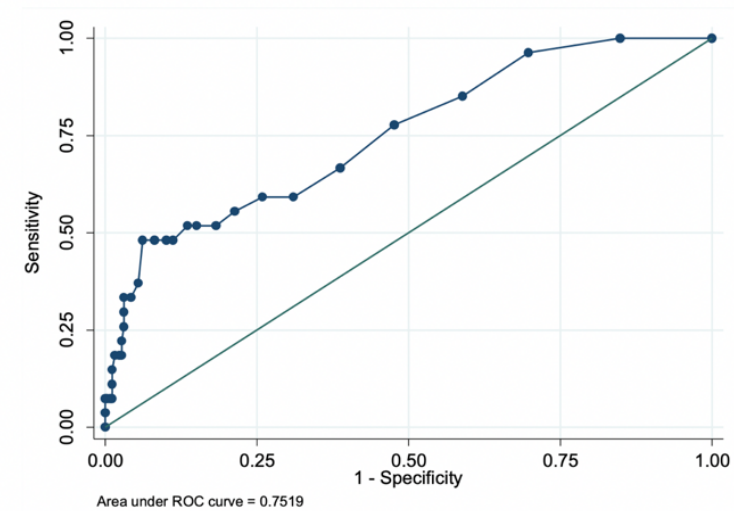

Figure 2. Calibration plot

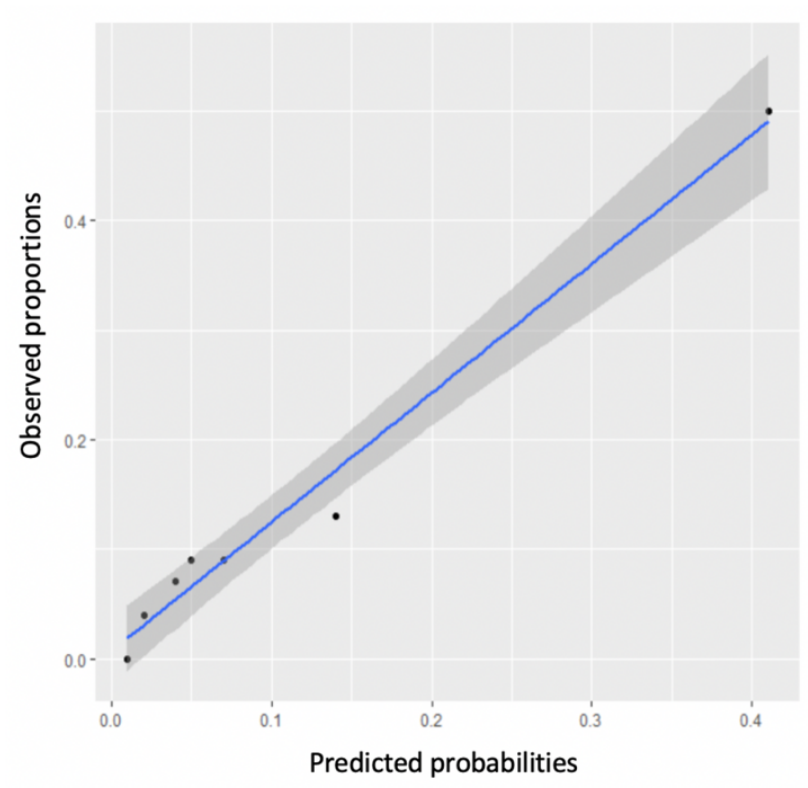




\section{Predictive factors of LNI and Briganti score inaccuracy}

On univariate analysis, median (range) serum PSA level for patients with and without LNI were respectively $11.1(9-22)$ and 7.5(5.4-10.8) $(p<0.001)$.

The proportion of high-risk patients was greater in the group with LNI $51.8 \%(\mathrm{~N}=14)$ vs $20.2 \%(\mathrm{~N}=52) ; \mathrm{p}<0.001)$. The proportion of cT3 was higher in the group with LNI $(11.1 \%(\mathrm{~N}=3)$ vs $0.8 \%(N=2) ; p<0.001)$. The rate of positive Briganti Score and the average Briganti Score were greater in the LNI group $(77.8 \%(\mathrm{~N}=21)$ and $19.3 \%$ vs $47.7 \%(\mathrm{~N}=123)$ and $6.3 \% ; \mathrm{p}<0.001)$.

The distribution of pathological stages was significantly different between patients with and without LNI (pT2: $11.1 \%(\mathrm{~N}=3)$ vs $62.4 \%(\mathrm{~N}=161)$; $\mathrm{pT3}: 85.2 \%(\mathrm{~N}=23)$ vs $36.8 \%(\mathrm{~N}=95)$; pT4: $3.7 \%(\mathrm{~N}=1)$ vs $0.4 \%(\mathrm{~N}=1) ; \mathrm{p}<0.001)$. The number of nodes removed was not statistically different between the two groups.

The proportion of adjuvant treatment was greater in the group of LNI patients $(37 \%(N=10)$ vs $10.1 \%(N=26) ; p<0.001)$.

Using multivariate logistic analysis, the PSA level (OR 4.9, 95\% $\mathrm{Cl} 2.1-11.8$ ), percentage of positives biopsies (OR 4.7, 95\% Cl 1.4-16) and T3 stage on MRI (OR 2.4, 95\% Cl 1-5.8) were independent predictive factors of LNI (Table 2). However, none of the factors studied proved predictive of Briganti score inaccuracy (Table 3).

\section{Biochemical recurrence-free survival}

5-year BCR-free survival rates were 74\% (IC95\%: 66\%-81\%) for intermediate-risk patients, and 70\% (IC95\%: 64\%-76\%) for the entire cohort. Figure 3 represents Kaplan-Meier curves for the BCR-free survival depending on LNI status, respectively for intermediate-risk patients (Figure 3a) and all patients (Figure 3b). BCR-free survival rate at 5 years was significantly lower for patients with LNI than those without LNI, for the intermediate-risk group as well as for the entire cohort (Intermediate-risk patients: $37 \%(I C 95 \%$ : $13 \%-62 \%)$ vs 77\%(IC95\%: 69\%-84\%), all patients: 33\%(IC95\%: 15\%- 22\%) vs 75\%(IC95\%: 68\%-80\%) ; Log rank test, $\mathrm{p}<0.05)$. 
Table 2. Predictive factors of lymph node metastases

\begin{tabular}{|c|c|c|c|}
\hline & & C95\%) & \\
\hline & $\begin{array}{l}\text { Univariate } \\
\text { analysis }\end{array}$ & $\begin{array}{l}\text { Multivariate } \\
\text { analysis }\end{array}$ & $p$ value \\
\hline PSA & & & \\
\hline$<10$ & 1 & 1 & \\
\hline$\geq 10$ & $4.1(1.8-9.5)$ & $4.9(2.1-11.8)$ & $<0.0001^{*}$ \\
\hline Clinical stage & & & \\
\hline $\mathrm{T} 1$ & 1 & - & \\
\hline $\mathrm{T} 2$ & $1.4(0.6-3.2)$ & - & \\
\hline T3 & $19.2(2.8-129.9)$ & - & \\
\hline Gleason score & & & \\
\hline $5-6$ & 1 & - & \\
\hline 7 & $2.4(0.8-7.2)$ & - & \\
\hline $8-9$ & $2.3(0.6-10.0)$ & - & \\
\hline $\begin{array}{l}\text { Percentage of pc } \\
\text { biopsies }\end{array}$ & & & \\
\hline$<33 \%$ & 1 & 1 & \\
\hline$\geq 33 \%-<66 \%$ & $3.1(1.1-8.3)$ & $3.6(1.3-10.4)$ & $0.016 *$ \\
\hline$\geq 66 \%$ & $5.2(1.6-16.7)$ & $4.7(1.4-16.0)$ & $0.013^{*}$ \\
\hline T3 stage on MRI & & & \\
\hline No & 1 & 1 & \\
\hline Yes & $2.9(1.3-6.6)$ & $2.4(1.0-5.8)$ & 0.046 \\
\hline $\mathrm{N}$ nodes remove & & & \\
\hline $1-4$ & 1 & - & \\
\hline $5-9$ & $0.8(0.2-3.2)$ & - & \\
\hline $10-19$ & $1.2(0.3-4.3)$ & - & \\
\hline$\geq 20$ & $0.8(0.1-5.0)$ & - & \\
\hline
\end{tabular}

Table 3. Potential predictive factors of Briganti score inaccuracy

\begin{tabular}{llll}
\hline & Adjusted OR & IC 95\% & p value \\
\hline $\begin{array}{l}\text { T3 stage on MRI } \\
\quad \text { No }\end{array}$ & 1.00 & & \\
$\quad$ Yes & 1.09 & $0.63-1.91$ & 0.750 \\
N biopsies performed & & & \\
$\quad \geq 12$ & 1.00 & & \\
$\quad<12$ & 0.92 & $0.56-1.52$ & 0.747 \\
Prostate volume & & & \\
$\quad \leq 30$ & 1.00 & & \\
$>30 ; \leq 80$ & 0.95 & $0.58-1.57$ & 0.855 \\
$>80$ & 1.18 & $0.32-4.33$ & 0.802 \\
\hline
\end{tabular}


Figure 3. Comparative survival curves between patients with and without lymph node invasion for intermediate-risk patients (3a. $\mathrm{N}=175)$ and the entire cohort (3b. $\mathrm{N}=285$ ) at 5 years

3a. Biochemical recurrence-free survival: Log rank test $\mathbf{=} 0.0001$

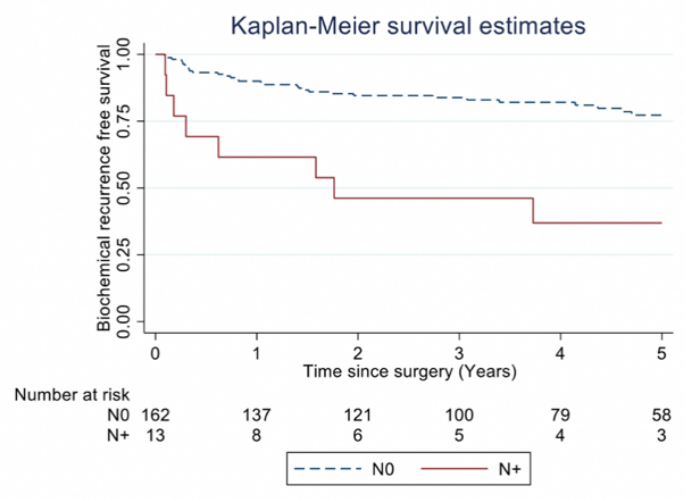

3b. Biochemical recurrence-free survival: Log rank test $\mathbf{= 0 . 0 0 0 0}$

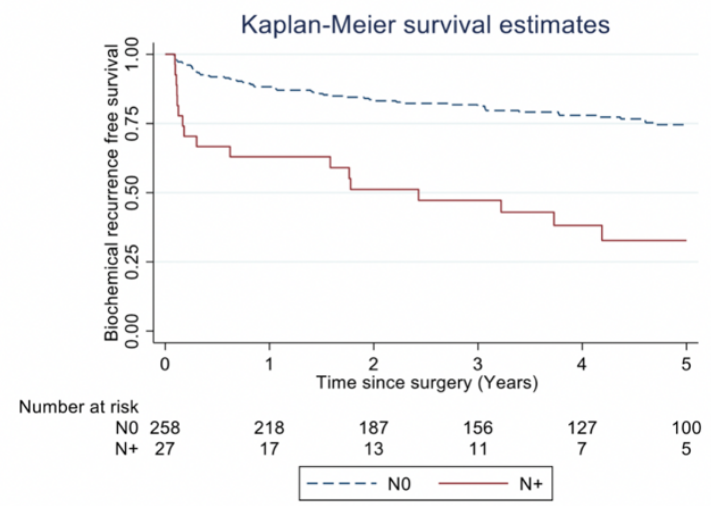

Consequences of the application of the Briganti nomogram

Figure 4 represents the distribution of patients according to the result of lymph node dissection and Briganti Score.

Figure 4. Results of Briganti Score application

4a. in intermediate-risk patients

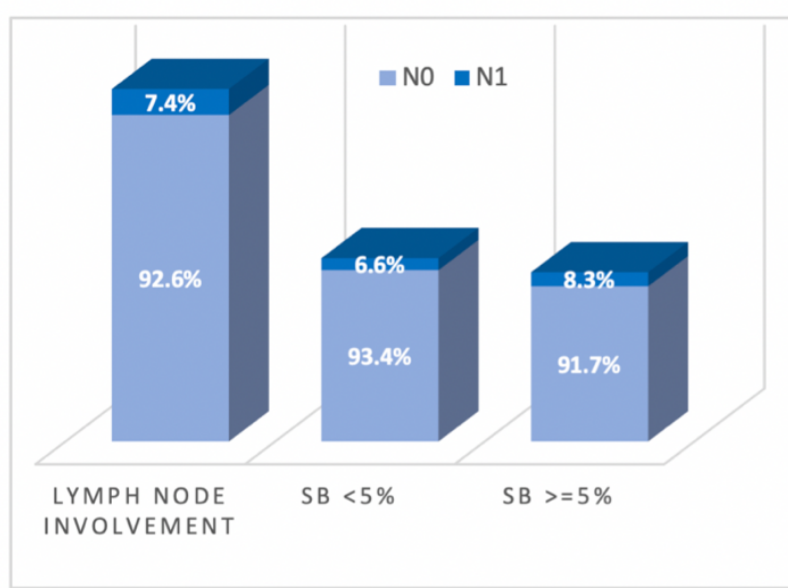

\begin{tabular}{|c|c|c|c|}
\hline & $\begin{array}{c}\text { Lymph node } \\
\text { involvement }\end{array}$ & SB $<5 \%$ & SB > = 5\% \\
\hline N1 & 13 & 6 & 7 \\
\hline N0 & 162 & 85 & 77 \\
\hline
\end{tabular}

4b. in the entire cohort

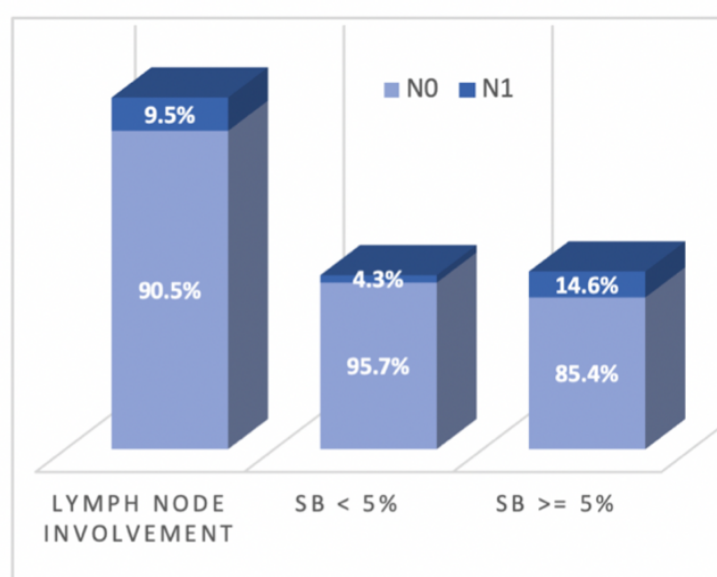

\begin{tabular}{|c|c|c|c|}
\hline & $\begin{array}{c}\text { Lymph node } \\
\text { involvement }\end{array}$ & SB $<5 \%$ & SB $>=5 \%$ \\
\hline N1 & 27 & 6 & 21 \\
\hline N0 & 258 & 135 & 123 \\
\hline
\end{tabular}


With a threshold of $5 \%$, focusing on intermediate-risk patients, $52 \%(\mathrm{~N}=91)$ had a Briganti score $<5 \% .6 .6 \%(\mathrm{~N}=6)$ of those patients had a LNI (Figure 4a). 141 (49.5\%) patients had a negative Briganti Score and among them 4.3\%(N=6) had a LNI (Figure $4 b$ ).

Among these patients with avoidable BPLND, the rate of complications $\geq$ Clavien 3 (including 1 death) was respectively $2.1 \%(\mathrm{~N}=6)$ and $2.3 \%(\mathrm{~N}=4)$.

In order to precise the clinical relevance of various thresholds, we analyzed the consequences of the application of a different threshold on the number of BPLNDs to perform in order to detect $1 \mathrm{LNI}$. The results summarizing the interest of the Briganti score according to the cut-off value used are shown in Table 4 . With a threshold set at $4 \% / 5 \%$, we would need to perform a total of 108/84 BPLNDs and detect 9/7 patients with LNI, resulting in 12 BPLNDs needed to detect $1 \mathrm{LNI}$ while missing 4 patients with LNI and a Briganti score under the threshold. Although reducing the overall number of BPLNDs performed, by increasing the threshold up to $7 \%$, the number of BPLNDs to perform to detect 1 patient with LNI would be 26, while missing 11 patients with LNI and a score under the threshold.

Table 4. Performance of the Briganti score according to the threshold chosen to discriminate patients with and without $\mathrm{LNI}$ - intermediate-risk patients

\begin{tabular}{llllllll}
\hline $\begin{array}{l}\text { Threshold } \\
\text { (\%) }\end{array}$ & \multicolumn{3}{c}{$\begin{array}{c}\text { BPLND not recommended } \\
\text { (below threshold) N(\%) }\end{array}$} & \multicolumn{3}{c}{$\begin{array}{c}\text { BPLND recommended } \\
\text { (above threshold) N(\%) }\end{array}$} & NPV \% (95\%Cl) \\
& All & LNI - & LNI+ & All & LNI - & LNI + & \\
\hline 4 & $67(38.3 \%)$ & $63(94.0 \%)$ & $4(6.0 \%)$ & $108(61.7 \%)$ & $99(91.7 \%)$ & $9(8.3 \%)$ & $94.0 \%(85.4-98.3)$ \\
5 & $91(52.0 \%)$ & $85(93.4 \%)$ & $6(6.6 \%)$ & $84(48.0 \%)$ & $77(91.7 \%)$ & $7(8.3 \%)$ & $93.4 \%(86.2-97.5)$ \\
6 & $110(62.9 \%)$ & $101(91.8 \%)$ & $9(8.2 \%)$ & $65(37.1 \%)$ & $61(93.8 \%)$ & $4(6.2 \%)$ & $91.8 \%(85.0-96.2)$ \\
7 & $123(70.3 \%)$ & $112(91.1 \%)$ & $11(8.9 \%)$ & $52(29.7 \%)$ & $50(96.2 \%)$ & $2(3.8 \%)$ & $91.1 \%(84.6-95.5)$ \\
\hline
\end{tabular}

\section{Discussion}

In this study, we report the results of its application on a separate cohort of 285 patients with localized prostate cancer including 175 intermediate-risk patients. The Briganti nomogram has previously been tested among patients of high risk group with an AUC between $65 \%$ and $72 \%[8,9,11,12]$. It has also been validated on cohorts of $D^{\prime}$ Amico all risk patients with an AUC between $79 \%$ and 83\% [13-15]. Nevertheless, Roumiguie and al [8] have more 
recently expressed reservations about the disappointing results they obtained with their group of intermediate-risk patients.

The accuracy of the Briganti score proved relevant for our entire cohort but insufficiently accurate for the population of interest, i.e. intermediate-risk patients, with respective AUC equal to $75.2 \%$ and $53.1 \%$. For comparison, Briganti et al. reported an AUC equal to $76.2 \%$ [6] and $87.6 \%$ [7] in their initial validation cohort. In our cohort composed mainly of intermediate-risk patients, median PSA level, proportion of clinical T3 and percentage of positive biopsies were close to those of the internal validation cohort of Briganti (respectively $7.8 \mathrm{ng} / \mathrm{ml}$ vs $6.3 \mathrm{ng} / \mathrm{ml}, 1.8 \%$ vs $5.3 \%$, and $36.1 \%$ vs $35.5 \%$ ) [7]. Of note, primary and secondary Gleason pattern >=4 was more frequently in our study (respectively $37.5 \%$ and $47.3 \%$ vs $17 \%$ and $31 \%$ ). The Briganti nomogram was validated using a cohort of patients diagnosed with extended biopsy protocols and BPLND. Indeed the number of total biopsy cores taken and lymph nodes retrieved in our study were lower (respectively 10 vs 19 and 12 vs 17) [7].

We acknowledge that the robot-assisted laparoscopic approach appeared, in this study, to retrieve a median number of lymph nodes inferior to the open approach, 9 (7-12) versus 11(8-16). These results are similar to other series of robotic BPLND $[13,16]$. This could be explained by learning curve of the supervising surgeon, and the involvement of junior surgeons, as outlined by a relatively high positive surgical margin rate. The lymph node retrieval technique and conditioning (separated into several flasks or monobloc) could also have influenced the number of nodes. Finally, the lymph node enumeration by the pathologist is subject to great inter-individual variability [17].

The morbidity rate of BPLND in this study was $9.1 \%$, lower than expected from previous publications. Indeed, series of eBPLND report complications rates varying from $10.5 \%$ to $19.8 \%$ [10]. A smaller number of nodes removed, consistent with a less-extensive dissection could be an explanation, although under-reporting of complications is also likely in this retrospective study. An attempt was made to evaluate the morbidity avoided if the score had been applied pre-operatively. For the entire cohort, $49.5 \%(\mathrm{~N}=141)$ of BPLND would have been omitted, preventing $2.1 \%(\mathrm{~N}=6)$ of complications $\geq$ Clavien 3 , including one death. For the intermediaterisk group, $52 \%(\mathrm{~N}=91)$ of avoidable BPLND and $2.3 \%(\mathrm{~N}=4)$ of complications $\geq$ Clavien 3 , including 1 death, would have been prevented. 
The PSA level and the percentage of positive biopsies were identified as predictors of $\mathrm{LNI}$ in our study. These results confirm those of the literature $[7,8,18]$. The percentage of positive biopsies also seems to be one of the key elements. Absent from the first nomogram in 2006, it was added in the calculation of the score in the updated version of 2012 based on the results of Heidenreich and al. $[7,18]$. Although not significant in our study probably due to lack of power explaining a wider confidence interval, the presence of T3 disease on MRI seems an interesting prognostic factor.

We report a BCR rate of $30.9 \%$ in this series mostly composed of intermediate and high-risk patients, probably linked with a rate of positive surgical margins on the high side of reported rates. Despite this, the 5-year BCR-free survival rates of our entire cohort and intermediate-risk, respectively equal to $70 \%$ and $74 \%$ were comparable to those of literature, probably thanks to prompt adjuvant or salvage treatment $[19,20]$.

The inaccuracy of the nomogram in our cohort was in part due to failure to detect patients with $\mathrm{LNI}$, but also because many patients with a Briganti score above $5 \%$ did not happen to have LNI. With a cut-off set at $5 \%$, half of intermediate-risk patients $(N=91)$ would have been spared BPLND and 6 LNI (6.6\%) would have been missed. Increasing the threshold to $6 \%$ or $7 \%$ would have resulted in a higher number of patients avoiding BPLND ( $N=110$ and 123 , respectively), with a good negative predictive value over $91 \%$ for this rare event, but looking at the number of BPLNDs needed to detect $1 \mathrm{LNI}$, the threshold of $5 \%$ proved more clinically relevant.

We must acknowledge many limitations of the present study. Its unicentric and retrospective character obviously limits the generalizability of its results. The long inclusion time over 12 years and a small number of patients included per year contributed to a lack of power, especially for subgroup analyses. We chose to report only BPLND-related complications as we believe these could have been prevented in patients with a low Briganti score, although it leads to an underestimation of the overall complication rate of the procedure. The relatively low median number of nodes retrieved, in part explained by the learning curves of the laparoscopic, and subsequently robotic techniques, could contribute to the lack of accuracy of the nomogram in this cohort, even though the proportion of patients with LNI seems in keeping with previously published studies [13-15]. This limitation had been identified during the setting-up phase of the study and we tried to mitigate it by providing the BCR-free survival curves for both cohorts with and without LNI identified at BPLND. The 
different survival profiles proved in favor of a limited contamination of the NO group. The use of a nomogram developed from a cohort including all risk categories on a subset of patients at intermediate risk could also explain the low accuracy obtained in this study. However, since BPLND is not recommended for low-risk patients, and should be performed for high-risk patients, it is toward this population that efforts to develop and validate prediction tools should be directed.

\section{Conclusion}

The Briganti nomogram applied to this cohort was not sufficiently accurate in predicting the risk of lymph node involvement in an intermediate-risk population. Still, its application before deciding to perform BPLND among intermediate-risk patients would have avoided 91 procedures (52\%) and failed to detect only 6 cases (6.6\%) of lymph node metastases. More precise tools are needed to precisely assess the LNI risk before surgery. The new version of a nomogram taking into account multiparametric MRI results could improve the accuracy and will also have to be validated.

\section{Acknowledgments}

Gaelle Fiard receives funding from and wishes to thank the Fondation de France and the European Urology Scholarship Program.

\section{Declarations}

\section{Funding}

Gaelle Fiard receives funding from and wishes to thank the Fondation de France and the European Urology Scholarship Program

\section{Conflicts of interest/Competing interests}

The authors declare that they have no conflict of interest.

Ethics approval

This retrospective study conducted from medical charts used data obtained during routine care. Data protection declaration was performed with the CNIL under number 2216943v0.

\section{Consent to participate}

Not applicable

Consent for publication

Not applicable

\section{Availability of data and material}

De-identified data will be made readily available to reviewers upon request 
Statistical software code will be made readily available to reviewers upon request

\section{Authors' contributions}

N Peilleron: Protocol/project development, Data collection or management, Manuscript writing/editing

A Seigneurin: Data analysis

C Herault: Data analysis

C Verry: Manuscript writing/editing

M Bolla: Manuscript writing/editing

JJ Rambeaud: Manuscript writing/editing

JL Descotes: Protocol/project development

JA Long: Manuscript writing/editing

G Fiard: Protocol/project development, Manuscript writing/editing

\section{References}

1. Schröder FH, Hugosson J, Roobol MJ, et al (2014) Screening and prostate cancer mortality: results of the European Randomised Study of Screening for Prostate Cancer (ERSPC) at 13 years of follow-up. The Lancet 384:2027-2035. https://doi.org/10.1016/S0140-6736(14)60525-0

2. Bill-Axelson A, Holmberg L, Garmo H, et al (2014) Radical Prostatectomy or Watchful Waiting in Early Prostate Cancer. N Engl J Med 370:932-942. https://doi.org/10.1056/NEJMoa1311593

3. Jones CU, Chetner MP, Rotman M (2011) Radiotherapy and Short-Term Androgen Deprivation for Localized Prostate Cancer. N Engl J Med 365(2):107-18. doi: 10.1056/NEJMoa1012348.

4. Rozet F, Hennequin C, Beauval J-B, et al (2016) Recommandations en onco-urologie 2016-2018 du CCAFU: Cancer de la prostate. Prog En Urol 27:S95-S143. https://doi.org/10.1016/S1166-7087(16)30705-9

5. Mottet N, Bellmunt J, Bolla M, et al (2017) EAU-ESTRO-SIOG Guidelines on Prostate Cancer. Part 1: Screening, Diagnosis, and Local Treatment with Curative Intent. Eur Urol 71:618-629. https://doi.org/10.1016/j.eururo.2016.08.003

6. Briganti A, Chun FK-H, Salonia A, et al (2006) Validation of a Nomogram Predicting the Probability of Lymph Node Invasion among Patients Undergoing Radical Prostatectomy and an Extended Pelvic Lymphadenectomy. Eur Urol 49:1019-1027. https://doi.org/10.1016/j.eururo.2006.01.043 
7. Briganti A, Larcher A, Abdollah F, et al (2012) Updated nomogram predicting lymph node invasion in patients with prostate cancer undergoing extended pelvic lymph node dissection: the essential importance of percentage of positive cores. Eur Urol 61:480487. https://doi.org/10.1016/j.eururo.2011.10.044

8. Roumiguié $M$, Beauval J-B, Filleron T, et al (2014) External validation of the Briganti nomogram to estimate the probability of specimen-confined disease in patients with high-risk prostate cancer. BJU Int 114:E113-119. https://doi.org/10.1111/bju.12763

9. Briganti A, Joniau S, Gontero P, et al (2012) Identifying the Best Candidate for Radical Prostatectomy Among Patients with High-Risk Prostate Cancer. Eur Urol 61:584-592. https://doi.org/10.1016/j.eururo.2011.11.043

10. Heidenreich A, Ohlmann CH, Polyakov S (2007) Anatomical Extent of Pelvic Lymphadenectomy in Patients Undergoing Radical Prostatectomy. Eur Urol 52:29-37. https://doi.org/10.1016/j.eururo.2007.04.020

11. Mearini L, Gacci M, Saleh O, et al (2014) External validation of nomogram predicting the probability of specimen-confined disease ( $p$ T2-3a, RONO) in patients undergoing radical prostatectomy and pelvic lymph node dissection. Urol Int 93:262-268. https://doi.org/10.1159/000354430

12. Hansen J, Becker A, Kluth LA, et al (2015) Assessing the clinical benefit of a nomogram to predict specimen-confined disease at radical prostatectomy in patients with high-risk prostate cancer: An external validation. Urol Oncol 33(9):384.e1-8. doi: 10.1016/j.urolonc.2015.02.017.

13. Dell'Oglio P, Abdollah F, Suardi N, et al (2014) External validation of the European association of urology recommendations for pelvic lymph node dissection in patients treated with robot-assisted radical prostatectomy. J Endourol 28:416-423. https://doi.org/10.1089/end.2013.0571

14. Gacci M, Schiavina R, Lanciotti M, et al (2013) External validation of the updated nomogram predicting lymph node invasion in patients with prostate cancer undergoing extended pelvic lymph node dissection. Urol Int 90:277-282. https://doi.org/10.1159/000343993

15. Hansen J, Rink M, Bianchi $M$, et al (2013) External validation of the updated Briganti nomogram to predict lymph node invasion in prostate cancer patients undergoing extended lymph node dissection. The Prostate 73:211-218. https://doi.org/10.1002/pros.22559

16. Gandaglia G, Sammon JD, Chang SL, et al (2014) Comparative Effectiveness of RobotAssisted and Open Radical Prostatectomy in the Postdissemination Era. J Clin Oncol 32:1419-1426. https://doi.org/10.1200/JCO.2013.53.5096

17. Sherbeck JP, Zhao L, Lieberman RW (2018) High Variability in Lymph Node Counts Among an International Cohort of Pathologists: Questioning the Scientific Validity of Node Counts. J Natl Compr Canc Netw 16:395-401. https://doi.org/10.6004/jnccn.2017.7064 
18. Heidenreich A, Pfister D, Thüer D, Brehmer B (2011) Percentage of positive biopsies predicts lymph node involvement in men with low-risk prostate cancer undergoing radical prostatectomy and extended pelvic lymphadenectomy. BJU Int 107:220-225. https://doi.org/10.1111/j.1464-410X.2010.09485.x

19. Beauval J-B, Cabarrou B, Gandaglia G, et al (2017) External validation of a nomogram for identification of pathologically favorable disease in intermediate risk prostate cancer patients. The Prostate 77:928-933. https://doi.org/10.1002/pros.23348

20. D'Amico AV, Whittington R, Malkowicz SB, et al (2002) Biochemical outcome after radical prostatectomy or external beam radiation therapy for patients with clinically localized prostate carcinoma in the prostate specific antigen era. Cancer 95:281-286. https://doi.org/10.1002/cncr.10657 